\title{
A split SUSY model from SUSY GUT
}

\section{Fei Wang, ${ }^{a, b}$ Wenyu Wang ${ }^{c}$ and Jin Min Yang ${ }^{b}$}

${ }^{a}$ Department of Physics and Engineering, Zhengzhou University, Zhengzhou 450000, P.R. China

${ }^{b}$ State Key Laboratory of Theoretical Physics, Institute of Theoretical Physics, Chinese Academy of Sciences, Beijing 100190, P.R. China

${ }^{c}$ Institute of Theoretical Physics, College of Applied Science, Beijing University of Technology, Beijing 100124, P.R. China

E-mail: feiwang@zzu.edu.cn, wywang@mail.itp.ac.cn, jmyang@itp.ac.cn

ABSTRACT: We propose to split the sparticle spectrum from the hierarchy between the GUT scale and the Planck scale. A split supersymmetric model, which gives non-universal gaugino masses, is built with proper high dimensional operators in the framework of $\mathrm{SO}(10)$ GUT. Based on a calculation of two-loop beta functions for gauge couplings (taking into account all weak scale threshold corrections), we check the gauge coupling unification and dark matter constraints (relic density and direct detections). We find that our scenario can achieve the gauge coupling unification and satisfy the dark matter constraints in some part of parameter space. We also examine the sensitivity of the future XENON1T experiment and find that the currently allowed parameter space in our scenario can be covered for a neutralino dark matter below about $1.0 \mathrm{TeV}$.

KeYWords: Supersymmetry Phenomenology

ArXiv EPRINT: 1501.02906 


\section{Contents}

1 Introduction $\quad 1$

2 A split SUSY model from SUSY GUT 2

3 Gauge coupling unification and dark matter constraints $\quad 6$

3.1 Inputs of our model 6

$\begin{array}{lll}3.2 & \text { The gauge coupling unification requirement } & 7\end{array}$

$\begin{array}{lll}3.3 & \text { Dark matter constraints } & 8\end{array}$

4 Conclusion $\quad 10$

$\begin{array}{ll}\text { A The two-loop beta function for gauge couplings } & 11\end{array}$

\section{Introduction}

The discovery of a $125 \mathrm{GeV}$ Higgs boson $[1,2]$ by both the ATLAS and CMS collaborations has completed the Standard Model (SM). The experimental data reported so far by LHC agree quite well with the SM predictions. On the other hand, the lack of significant hints in electroweak precision tests and the absence of evidences for new particle contents at the LHC challenge many proposals of new physics beyond the SM, including weak scale supersymmetry (SUSY).

SUSY, which was regarded for a long time as one of the most appealing extensions of the SM, has many desirable features. For example, the observed $125 \mathrm{GeV}$ Higgs boson falls within the narrow $115-135 \mathrm{GeV}$ window predicted by the minimal supersymmetric standard model (MSSM). Besides, the genuine unification of gauge couplings [3-9], which can hardly be achieved in the SM, can be successfully realized in the framework of low energy SUSY. Also, the puzzle of cosmic dark matter can naturally be explained in SUSY.

Although SUSY is appealing, null search results of sparticles at LHC suggest that either low energy SUSY needs to be tuned or sparticles are well above the weak scale. In fact, the LHC data has already set a limit [10-13] $m_{\tilde{g}}>1.5 \mathrm{TeV}$ for $m_{\tilde{q}} \sim m_{\tilde{g}}$ and $m_{\tilde{g}} \gtrsim 1 \mathrm{TeV}$ for $m_{\tilde{q}} \gg m_{\tilde{g}}$ for certain popular CMSSM models. On the other hand, the observed mass of the Higgs boson requires rather large loop effects of top squarks in the MSSM and CMSSM, which implies some extent of fine-tuning [14, 15]. So naturalness in SUSY may be realized in a more involved way even though it was initially proposed to solve the hierarchy problem.

Split SUSY, proposed in [16-18], gives up the naturalness criterion while keeps the other two main advantages: the gauge coupling unification and viable dark matter candidates. This scenario assumes a very high scalar mass scale $M_{S}$ and the low energy 
spectrum contains only the gauginos and higgsinos as well as a fine-tuned Higgs boson in addition to the SM sector. The SUSY CP and flavor problems can naturally be solved in this scenario due to very heavy sfermions. The latest results of the Higgs mass from the LHC measurement, together with the requirement of gauge coupling unification, suggest a scalar superpartner mass scale roughly of order $M_{S} \sim 100-1000 \mathrm{TeV}$ [19-21], which indicates a moderately split spectrum for split SUSY. The hierarchy of sparticle spectrum is argued to be determined by the gauge loop factor [20]. We propose in this paper to split the sparticle spectrum from the hierarchy between the GUT scale and the Planck scale.

Actually, there are various ways to split the sparticle spectrum. In this work we propose to use the generalized gravity mediation [22-33] with non-renormalizable Kähler potential and superpotential. Because GUT is one of the retained motivations of split SUSY, certain high-representation Higgs fields of GUT group could appear in the non-renormalizable Kähler potential and superpotential. Such high-representation fields could not only amend the gauge coupling unification condition at the GUT scale, but also establish new relations among various theory inputs. In our scenario we have non-universal gaugino masses at the GUT scale (for other scenarios giving non-universal gaugino masses, see [34-44]). Such nonuniversal gaugino masses can naturally appear with non-renormalizable Kähler potential and superpotential involving various high-representation Higgs fields.

This paper is organized as follows. In section 2 we present our model. In section 3 we check the phenomenology of our model. Based on a calculation of two-loop beta functions for gauge couplings (taking into account all weak scale threshold corrections), we check the gauge coupling unification and the dark matter constraints. The future XENON1T sensitivity to our scenario is also examined. Finally, in section 4 we give our conclusions.

\section{A split SUSY model from SUSY GUT}

There are many possible ways to mediate the SUSY breaking effects from the hidden sector to the visible sector. A very interesting and predictive possibility is the gravity mediation. With certain non-renormalizable terms, proper soft SUSY breaking parameters can be generated. In many popular gravity mediation scenarios, the Kähler potential is assumed to be minimal. However, a general Kähler potential seems to be more natural. When certain high-representation chiral fields for the GUT group are involved in the non-renormalizable Kähler potential, the kinetic terms of superfields could have other contributions after the GUT symmetry breaking. New non-renormalizable terms in the superpotential involving high-representation fields could also be important.

In general, the non-vanishing F-term VEVs of certain fields which break SUSY could be either gauge singlets or non-singlets. In this section, we propose that SUSY breaking is triggered by the GUT group non-singlet F-term VEVs. The gaugino and sfermion masses will be generated by some non-renormalizable operators which could arise from integrating out certain gravitational effects. In order to get more compact spectrum and simplify the relevant expressions, we adopt the SO(10) GUT group with the Georgi-Glashw SU(5) GUT group as an intermediate stage in the symmetry breaking chain:

$$
\mathrm{SO}(10) \frac{}{\mathbf{1 6}, \overline{\mathbf{1 6}}} \mathrm{SU}(5) \times \mathrm{U}(1)_{X} \frac{}{\mathbf{5 4}(\mathbf{4 5})} \mathrm{SU}(3)_{c} \times \mathrm{SU}(2)_{L} \times \mathrm{U}(1)_{Y}
$$


Here $\mathrm{U}(1)_{X}$ is broken by possible $\nu_{L}^{c}$ component VEVs of $H_{16}$ and $\bar{H}_{\overline{\mathbf{1 6}}}$ Higgs fields. Therefore, most of the results in this paper are also valid in $\mathrm{SU}(5)$ GUT. The possibility of $\mathrm{SO}(10)$ to Pati-Salam will be considered elsewhere.

In order to accommodate the non-minimal Kähler potential, we need to know the group products of various $\mathrm{SO}(10)$ representations [45]. The spinor representation (for the matter part) can be decomposed as

$$
\overline{16} \otimes 16=1 \oplus 45 \oplus \mathbf{2 1 0},
$$

while the adjoint representation for gaugino is

$$
(45 \otimes \mathbf{4 5})_{\text {symmetric }}=\mathbf{1} \oplus \mathbf{5 4} \oplus \mathbf{2 1 0} \oplus \mathbf{7 7 0} .
$$

and the fundamental representation of Higgs is

$$
10 \otimes 10=1 \oplus 45 \oplus 54 .
$$

We assume the non-minimal gauge kinetic term for vector supermultiplets

$$
\mathcal{L}=\int d^{2} \theta W^{\alpha}\left(\delta_{\alpha \beta}+\eta \frac{\Phi_{\alpha \beta}}{M_{*}}\right) W^{\beta} .
$$

with $M_{*}$ being the reduced Planck scale. So the kinetic part for gauge field with nonminimal Kähler potential is given by

$$
\mathcal{L}=-\frac{1}{4 k} \operatorname{Tr}\left[F_{\mu \nu} F^{\mu \nu}\right]-\frac{\eta}{4 k M_{*}} \operatorname{Tr}\left[F_{\mu \nu} \Phi F^{\mu \nu}\right]
$$

with $k$ being the normalization factor for various representations according to $\operatorname{Tr}\left(T^{a} T^{b}\right)=$ $k \delta^{a b}$. After the GUT non-singlet develops a VEV, $\langle\Phi\rangle=v+F_{\Phi} \theta^{2}$ with $v^{2} \gg F_{\Phi}$, the unification condition turns into

$$
g_{1}^{2}\left(M_{X}\right)\left(1+\frac{\eta v}{M_{*}} \delta_{1}\right)=g_{2}^{2}\left(M_{X}\right)\left(1+\frac{\eta v}{M_{*}} \delta_{2}\right)=g_{3}^{2}\left(M_{X}\right)\left(1+\frac{\eta v}{M_{*}} \delta_{3}\right)
$$

with $\delta_{3,2,1}$ being the appropriate group factors for $\mathrm{SU}(3)_{c}, \mathrm{SU}(2)_{L}, \mathrm{U}(1)_{Y}$, respectively. If the hierarchy between the (first step Georgi-Glashow SU(5)) GUT scale and the Planck scale is not small, the previous GUT conditions turn into two independent new GUT conditions:

$$
\begin{gathered}
F_{1} \equiv \frac{\frac{g_{1}^{2}}{g_{2}^{2}}\left(M_{X}\right)-1}{\frac{g_{1}^{2}}{g_{3}^{2}}\left(M_{X}\right)-1}=\frac{\delta_{2}-\delta_{1}}{\delta_{3}-\delta_{1}}=-\frac{2}{3}, \\
F_{2} \equiv \frac{\frac{g_{2}^{2}}{g_{1}^{2}}\left(M_{X}\right)-1}{\frac{g_{2}^{2}}{g_{3}^{2}}\left(M_{X}\right)-1}=\frac{\delta_{1}-\delta_{2}}{\delta_{3}-\delta_{2}}=\frac{2}{5} .
\end{gathered}
$$

For non-singlet $\Phi$, the F-term $F_{\Phi}$ can be decomposed as $\left(F_{\Phi}\right)_{a b}=F_{U} \cdot A_{a b}$ with $A_{a b}$ being the group factor and $F_{U}$ the universal part. The review of the group structure can be found in [45]. 
The group structure of the $\mathbf{2 4}$ component F-term VEV of $\mathbf{5 4}$ representation Higgs can be written in terms of $10 \times 10$ matrix

$$
\left\langle F_{\mathbf{5 4}}\right\rangle_{a b}=F_{\mathbf{5 4}}^{U} A_{a b}=F_{\mathbf{5 4}}^{U} \sqrt{\frac{3}{5}}\left(\frac{1}{3}, \frac{1}{3}, \frac{1}{3},-\frac{1}{2},-\frac{1}{2}, \frac{1}{3}, \frac{1}{3}, \frac{1}{3},-\frac{1}{2},-\frac{1}{2}\right) .
$$

The gaugino will get contribution from

$$
\mathcal{L} \supseteq \frac{<F_{\Phi}>_{\alpha \beta}}{M_{*}} \lambda^{\alpha} \lambda^{\beta}
$$

with the F-term VEVs $\left(F_{\mathbf{5 4}}\right)_{a b}=F_{\mathbf{5 4}}^{U} \cdot A_{a b}$. Here the universal part $F_{\mathbf{5 4}}^{U}$ is independent of the group structure. Then the non-universal gaugino masses are given by

$$
M_{1}=-\frac{1}{6} \sqrt{\frac{3}{5}} m_{1 / 2}, \quad M_{2}=-\frac{1}{2} \sqrt{\frac{3}{5}} m_{1 / 2}, \quad M_{3}=\frac{1}{3} \sqrt{\frac{3}{5}} m_{1 / 2}
$$

with

$$
m_{1 / 2}=\frac{F_{54}^{U}}{M_{*}} .
$$

The sfermion masses and kinetic term will be generated by the following non-renormalizable Kähler potential

$$
K=\frac{1}{M_{*}^{2}} \phi_{a}^{\dagger}\left(\Phi^{\dagger} \Phi\right)_{a b} \phi_{b}
$$

with proper F-term and lowest component VEVs of $\Phi$, respectively. Here we assume that the universal part of the kinetic terms $\Phi^{\dagger} \Phi$ is approximately canceled by a similar high dimensional operators with a lowest component VEV of singlet.

We know from the group theory that possible contributions to Kähler potential for matter content (filled in $\mathbf{1 6}$ representation of $\mathrm{SO}(10)$ ) can arise from the following type of Higgs fields

$$
1 \oplus 45 \supset 54 \otimes 54, \quad 1 \oplus \mathbf{2 1 0} \supset \overline{\mathbf{1 6}} \otimes 16 .
$$

So the F-term VEV of the $\mathbf{5 4}$ representation chiral superfield will contribute to nonuniversal sfermion masses of order $\left(F_{54} / M_{*}\right)^{2}$ in addition to possible contributions from $F_{16}$. We assume $F_{16} \sim F_{54}$ and we always have $F_{\overline{16}}=F_{16}$. Since $v_{16} \gg v_{54}$, the 54 representation field will give sub-leading contributions and we will not include them explicitly in the following expressions. From the group structure there are several possible contractions for matter fields $\phi$ with the form of Kähler potential

$$
\begin{aligned}
K \supset & \frac{1}{M_{*}^{2}} \sum_{a=1}^{3}\left(\phi_{a, \mathbf{1 6}}^{\dagger} \otimes \phi_{a, \mathbf{1 6}}\right)^{\mathbf{1}}\left[\left(d_{\mathbf{1}} \Phi_{H_{\mathbf{1 6}}}^{\dagger}+\tilde{d}_{\mathbf{1}} \Phi_{\bar{H}_{\overline{\mathbf{1 6}}}}\right) \otimes\left(f_{\mathbf{1}} \Phi_{H_{\mathbf{1 6}}}+\tilde{f}_{\mathbf{1}} \Phi_{\bar{H}_{\overline{\mathbf{1 6}}}}^{\dagger}\right)\right]^{\mathbf{1}} \\
& +\frac{1}{M_{*}^{2}} \sum_{a=1}^{3}\left(\phi_{a, \mathbf{1 6}}^{\dagger} \otimes \phi_{a, \mathbf{1 6}}\right)_{m n}^{\mathbf{4 5}}\left[\left(d_{\mathbf{4 5}} \Phi_{H_{\mathbf{1 6}}}^{\dagger}+\tilde{d}_{\mathbf{4 5}} \Phi_{\bar{H}_{\overline{\mathbf{1 6}}}}\right) \otimes\left(f_{\mathbf{4 5}} \Phi_{H_{16}}+\tilde{f}_{\mathbf{4 5}} \Phi_{\bar{H}_{\overline{\mathbf{1 6}}}}^{\dagger}\right)\right]_{m n}^{\mathbf{4 5}} \quad(2.15) \\
& +\frac{1}{M_{*}^{2}} \sum_{a=1}^{3}\left(\phi_{a, \mathbf{1 6}}^{\dagger} \otimes \phi_{a, \mathbf{1 6}}\right)_{m n l p}^{\mathbf{2 1 0}}\left[\left(d_{\mathbf{2 1 0}} \Phi_{H_{16}}^{\dagger}+\tilde{d}_{\mathbf{2 1 0}} \Phi_{\bar{H}_{\overline{16}}}\right) \otimes\left(f_{\mathbf{2 1 0}} \Phi_{H_{16}}+\tilde{f}_{\mathbf{2 1 0}} \Phi_{\bar{H}_{\overline{\mathbf{1 6}}}}^{\dagger}\right)\right]_{m n l p}^{\mathbf{2 1 0}}
\end{aligned}
$$

where $d, f, \tilde{d}, \tilde{f}$ denote the corresponding combination coefficients and $a$ is the family index. 
It can be checked that only the first term in eq. (2.15) contributes in this scenario. Then the soft sfermion masses are given by

$$
\epsilon^{2} \tilde{m}_{16}^{2}=d_{1} f_{1} \frac{F_{16}^{*} F_{16}}{M_{*}^{2}}+\tilde{d}_{1} \tilde{f}_{1} \frac{F_{16}^{*} F_{\overline{16}}}{M_{*}^{2}}+d_{1} \tilde{f}_{1} \frac{F_{16}^{*} F_{\overline{16}}}{M_{*}^{2}}+\tilde{d}_{1} f_{1} \frac{F_{16}^{*} F_{16}}{M_{*}^{2}}
$$

after we take into account the normalization factor $\epsilon^{2}=v_{\mathbf{1 6}}^{2} / M_{*}^{2}$ of the kinetic term. So we have

$$
\tilde{m}_{16}^{2} \sim \frac{F_{16}^{*} F_{16}}{M_{*}^{2} \epsilon^{2}} \sim \frac{F_{16}^{*} F_{16}}{v_{16}^{2}} .
$$

We can see that the typical gaugino mass scale $m_{1 / 2}$ is suppressed by a factor $\epsilon$ relative to the sfermion mass scale.

The soft SUSY breaking masses for the Higgs potential can be similarly obtained

$$
\begin{aligned}
K \supset & \frac{1}{M_{*}^{2}}\left(\phi_{10}^{\dagger} \otimes \phi_{10}\right)^{\mathbf{1}}\left[\left(g_{\mathbf{1}} \Phi_{H_{16}}^{\dagger}+\tilde{g}_{1} \Phi_{\bar{H}_{\overline{16}}}\right) \otimes\left(h_{\mathbf{1}} \Phi_{H_{16}}+\tilde{h}_{\mathbf{1}} \Phi_{\bar{H}_{\overline{16}}}^{\dagger}\right)\right]^{\mathbf{1}} \\
& +\frac{1}{M_{*}^{2}}\left(\phi_{\mathbf{1 0}}^{\dagger} \otimes \phi_{\mathbf{1 0}}\right)_{m n}^{45}\left[\left(g_{45} \Phi_{H_{16}}^{\dagger}+\tilde{g}_{\mathbf{4 5}} \Phi_{\bar{H}_{\overline{16}}}\right) \otimes\left(h_{\mathbf{4 5}} \Phi_{H_{16}}+\tilde{h}_{\mathbf{4 5}} \Phi_{\bar{H}_{\overline{16}}}^{\dagger}\right)\right]_{m n}^{45},
\end{aligned}
$$

with also contributions from $F_{\mathbf{5 4}}$ after we add similar terms involving the $\mathbf{5 4}$ representation Higgs fields. Both contributions are at the same order and the soft SUSY breaking Higgs masses are given by $m_{H_{u, d}}^{2} \sim\left(m_{1 / 2}\right)^{2}$. Thus we can see that the soft SUSY breaking Higgs mass parameters can be at the same order as the gaugino.

The trilinear terms will also get contributions from both $\mathbf{1 6}$ and $\overline{\mathbf{1 6}}$ representation Higgs fields. The relevant non-renormalizable superpotential has the form

$$
\begin{aligned}
W \supset & \frac{y_{\text {Yukawa }}}{M_{*}^{2}}\left(\Phi_{\bar{H}_{\overline{\mathbf{1 6}}}} \otimes \Phi_{H_{\mathbf{1 6}}}\right)^{\mathbf{1}}\left[C^{\mathbf{1}}\left(\mathbf{1 6} \mathbf{6}_{\mathbf{i}} \otimes \mathbf{1 6} \mathbf{6}_{\mathbf{i}}\right)_{\mathbf{1 0}}^{m} \mathbf{1 0}^{m}\right] \\
& +\frac{y_{\text {Yukawa }}}{M_{*}^{2}}\left(\Phi_{\bar{H}_{\overline{16}}} \otimes \Phi_{H_{16} \mathbf{6}}\right)_{m n}^{\mathbf{4 5}}\left[C^{\mathbf{4 5}}\left(\mathbf{1 6}_{\mathbf{i}} \otimes \mathbf{1 6}_{\mathbf{i}}\right)_{\mathbf{1 2 0}}^{m n l} \mathbf{1 0}^{l}\right] \\
& +\frac{y_{\text {Yukawa }}}{M_{*}^{2}}\left(\Phi_{\bar{H}_{\overline{\mathbf{1 6}}}} \otimes \Phi_{H_{16}}\right)_{m n l p}^{\mathbf{2 1 0}}\left[C^{\mathbf{2 1 0}}\left(\mathbf{1 6}_{\mathbf{i}} \otimes \mathbf{1 6}_{\mathbf{i}}\right)_{\mathbf{1 2 6}}^{m n l p q} \mathbf{1 0}{ }^{q}\right] .
\end{aligned}
$$

Again, here only the first term contributes. From the lowest component VEV and F-term VEV of $H_{16}$ and $H_{\overline{16}}$, we can obtain the trilinear coupling as

$$
\epsilon^{2} A_{y}=C^{\mathbf{1}}\left[\frac{v_{\mathbf{1 6}} F_{\overline{\mathbf{1 6}}}}{M_{*}^{2}}+\frac{v_{\overline{16}} F_{\mathbf{1 6}}}{M_{*}^{2}}\right] y_{\text {Yukawa }} .
$$

after we normalize the kinetic term for the $\mathbf{1 6}$ representation matter contents. Thus, we see that

$$
A_{0}=\frac{A_{y}}{y_{\text {Yukawa }}} \sim \frac{F_{\overline{16}}}{v_{16}}
$$

is typically of the same scale as sfermion masses.

The $B_{\mu}$-term and $\mu$ term are given by

$$
W \supset\left(M+\Phi_{45}\right) \phi_{10} \phi_{10}+k_{1}\left(M+\Phi_{45}\right) \frac{\left(\Phi_{54}\right)}{M_{*}} \phi_{10} \phi_{10} .
$$


We will not discuss in detail the doublet-triplet splitting problem in GUT and just use the fact that the combination $M+\left\langle\Phi_{45}\right\rangle$ will lead to (by possible tuning or some mechanism) proper low energy effective $\mu$ term.

The $B_{\mu}$-term will be generated after $\Phi_{54}$ acquires F-term VEVs

$$
B_{\mu}=-k_{1} \sqrt{\frac{3}{5}} \frac{F_{54}^{U}}{M_{*}} \mu .
$$

The electroweak symmetry breaking condition can relate different parameters as

$$
\begin{aligned}
|\mu|^{2} & =-\frac{M_{Z}^{2}}{2}+\frac{1}{\tan ^{2} \beta-1}\left(m_{H_{d}}^{2}-\tan ^{2} \beta m_{H_{u}}^{2}\right), \\
2 B_{\mu} & =\sin 2 \beta\left(m_{H_{d}}^{2}+m_{H_{u}}^{2}+2|\mu|^{2}\right) .
\end{aligned}
$$

Although there are many possibilities for the choice of $\mu$ from electroweak symmetry breaking condition, the relation eq. (2.23) will further constrain the choice of $\mu$. Combining eq. (2.23) with the (RGE modified) approximation $m_{H_{d}}^{2} \gtrsim m_{H_{u}}^{2}$, we can see that the symmetry breaking condition requires $|\mu|^{2} \sim-m_{H_{u}}^{2} \sim B_{\mu} \sim m_{1 / 2}^{2}$ for a negative $m_{H_{u}}^{2}$. For a positive $m_{H_{u}}^{2}$, it is difficult to reconcile a large $\tan \beta$ and $m_{H_{d}}^{2} \gtrsim m_{H_{u}}^{2}$ in case of $\mu \ll m_{h_{u}}^{2}$. Only if the RGE running can affect greatly the GUT scale relation $m_{H_{d}}^{2}=m_{H_{u}}^{2}$ can a relatively heavy $\mu$ be possible. The cases of $\tan \beta \sim 1$ which indicate $|\mu|^{2} \gg\left|m_{h_{d}}^{2}\right|$ for both signs of $m_{H_{u}}^{2}$ can hardly be compatible with eq. (2.23).

So we see that in our scenario the hierarchy between the GUT scale and the Planck scale is used in splitting the SUSY soft spectrum in contrast to the gauge loop factor appeared in simply unnatural supersymmetry [20].

\section{Gauge coupling unification and dark matter constraints}

\subsection{Inputs of our model}

In this section we check the gauge coupling unification and dark matter constraints in our scenario. From the previous section, we can see the inputs of our scenario at the GUT scale:

- The gaugino masses with the raito

$$
M_{1}: M_{2}: M_{3}=-1:-3: 2
$$

- The hierarchy between the GUT scale (SO(10) breaking scale) and the Planck scale $\epsilon$.

- The higgsino mass $\mu$ which should be at the same sacle as gaugino mass.

- The parameter $\tan \beta$. We scan it in the range $1 \sim 50$.

The sfermion mass $m_{0}$, determined by $m_{1 / 2} / \epsilon$, and the trilinear term $A_{0} \simeq m_{0}$ are not independent parameters in our model. This scenario predicts different parameter values in comparison to the universal gaugino mass scenario [21]. It is well known that the ratios of 
gaugino masses and the corresponding gauge couplings in SUSY are RGE-invariant up to one-loop level

$$
\frac{d}{d \ln \mu}\left(\frac{M_{i}}{g_{i}^{2}}\right)=0
$$

If we assume universal gaugino masses $M_{1}=M_{2}=M_{3} \equiv M_{U}$ at the GUT scale, from the RGE-invariant ratio

$$
\frac{M_{1}}{g_{1}^{2}}=\frac{M_{2}}{g_{2}^{2}}=\frac{M_{3}}{g_{3}^{2}}=\frac{M_{U}}{g_{U}^{2}}
$$

and the electroweak scale inputs we can have a mass relation given by

$$
M_{1}: M_{2}: M_{3} \simeq 1: 2: 6
$$

at electroweak scale. On the other hand, if we assume non-universal gaugino masses at the GUT scale, the above gaugino mass ratios at the electroweak scale will be approximately changed to

$$
M_{1}: M_{2}: M_{3} \simeq 1: 6:-12 .
$$

We should note again that the formula eq. (3.1) can no longer be valid below $M_{S}$ where heavy scalars decouple. So the exact gaugino mass ratio in split SUSY should be obtained by the subsequent RGE running from $M_{S}$ to EW scale.

\subsection{The gauge coupling unification requirement}

We study the gauge coupling unification with the two-loop RGE running of gauge couplings, taking into account all weak scale threshold corrections. The relevant analytical results for the two-loop beta functions are given in the appendix. It is well known that the two-loop RGE running for gauge couplings are scheme independent [46], so we use the $\overline{M S}$ couplings in our study of the gauge coupling unification. Lacking the full knowledge responsible for GUT symmetry breaking, especially the mechanism used to solve the doublet-triplet splitting problem which is model dependent, we therefore neglect such GUT scale threshold corrections in our study. In order to make our calculation reliable, the first step SU(5) GUT scale must be much lower than the Planck scale so that the gravitational effects can be neglected. Besides, the GUT scale can not be too low, otherwise it will lead to fast proton decay. The constraint is $[21,47-50]$

$$
1.0 \times 10^{19} \mathrm{GeV}>M_{\mathrm{GUT}}>\sqrt{35 \alpha_{\mathrm{GUT}}}\left(6.9 \times 10^{15}\right) \mathrm{GeV} .
$$

In our numerical study we input the central values of $g_{1}$ and $g_{2}$ while for $g_{3}$ we require it in the $3 \sigma$ at the electroweak scale. Other inputs at the electroweak scale, for example, the top Yukawa coupling $y_{t}$, are extracted from the SM taking into account the threshold corrections. Relevant details can be seen in the appendix of $[21,51,52]$. Because of the uncertainty of the GUT scale threshold contributions, we adopt the criteria that the gauge coupling unification is satisfied when the three couplings differ within the range $<0.005$. 


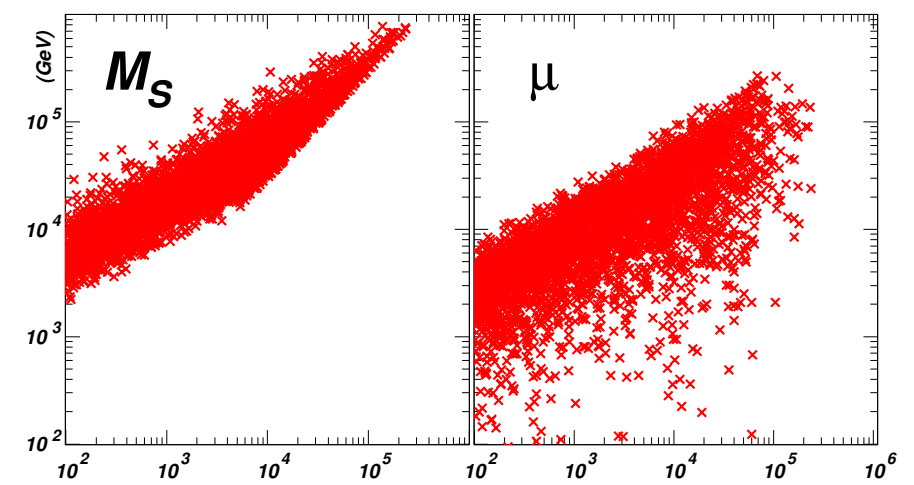

$M_{2}(\mathrm{GeV})$

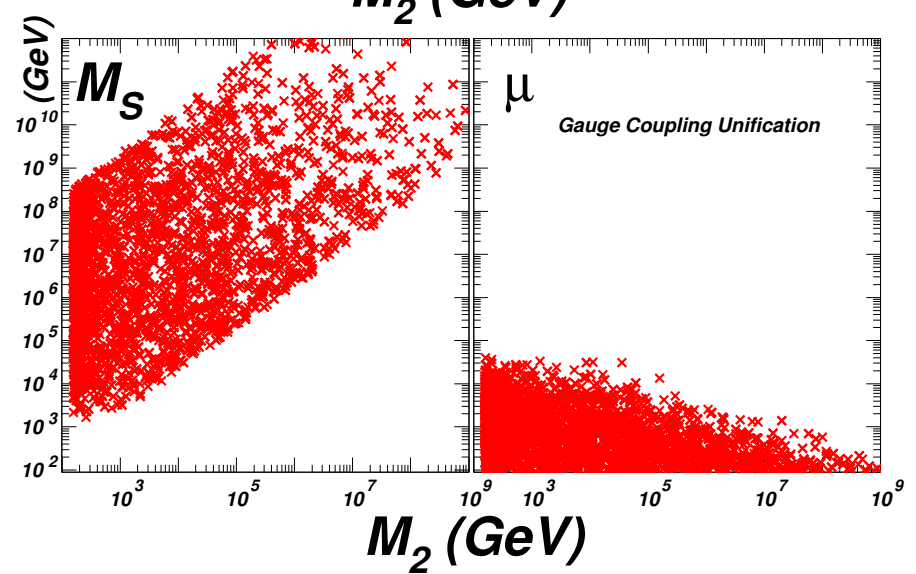

Figure 1. The scatter plots of the parameter space with gauge coupling unification in case of universal gaugino input (upper) and non-universal gaugino input (lower) at the GUT scale.

Figure 1 shows the result of the parameter space $\left(M_{2}, M_{S}, \mu\right)$ with successful gauge coupling unification in case of universal condition eq. (3.3) and non-universal condition eq. (3.4), respectively. We can see that the gaugino unification gives a stringent constraint on the parameter space. From the upper-left panel we can find an upper bound for $M_{S}$, which is about $10^{6} \mathrm{GeV}$. Since split SUSY requires $M_{S} \gg M_{\tilde{g}_{i}}$, we can also obtain an upper bound for $M_{2}$ correspondingly. From the upper-right panel we can find upper limits for $\mu$ and $M_{2}$, which are around $100 \mathrm{TeV}$, independent of the $M_{S}$ value. However, the constraints for the non-universal gaugino scenario are rather mild. From the lower panels, we can see that $M_{S}$ can be as high as $10^{12} \mathrm{GeV}$ while $M_{2}$ can be $10^{9} \mathrm{GeV}$. The $\mu$ parameter, which plays an important role in gauge coupling unification, also has an upper bound around $10^{5} \mathrm{GeV}$ for both non-universal and universal case.

\subsection{Dark matter constraints}

Now we study the dark matter constraints in our scenario, using the latest dark matter relic density data from Planck [53], WMAP [54] and the direct detection limits from XENON100 [55] and the LUX [56]. The package DarkSUSY [57] is used to scan the parameter space of our scenario. In addition to the inputs defined above, we also require $1<\tan \beta<50$. In order to calculate the relic density of dark matter, we use the fact 
that the effects of heavy sfermions and heavy Higgs fields almost entirely decouple when $M_{S}=M_{A}>5 \mathrm{TeV}$ [58]. So in our numerical study, we keep the samples which satisfy the GUT constraints and then set $M_{S}=M_{A}=10 \mathrm{TeV}$ in DarkSUSY to carry out the dark matter related calculations.

For illustration we set $M_{S}=30,50,100,200 \mathrm{TeV}$ and show the allowed ranges for the parameters $M_{2}, \mu, \tan \beta$. In our scan, we take into account the current dark matter and collider constraints (the details can be found in our previous work [21]):

(1) We require that the relic density of the neutralino dark matter satisfies the Planck result $\Omega_{D M}=0.1199 \pm 0.0027$ [53] (in combination with the WMAP data [54]).

(2) The LEP lower bounds on neutralino and charginos, including the invisible decay of $Z$-boson. For LEP experiments, the most stringent constraints come from the chargino mass and the invisible $Z$-boson decay. We require $m_{\tilde{\chi}^{ \pm}}>103 \mathrm{GeV}$ and the invisible decay width $\Gamma\left(Z \rightarrow \tilde{\chi}_{0} \tilde{\chi}_{0}\right)<1.71 \mathrm{MeV}$, which is consistent with the $2 \sigma$ precision EW measurement $\Gamma_{\text {inv }}^{\text {non-SM }}<2.0 \mathrm{MeV}$.

(3) The precision electroweak measurements. We require the oblique parameters [59, 60] to be compatible with the LEP/SLD data at $2 \sigma$ confidence level [61].

(4) The combined mass range for the Higgs boson: $123 \mathrm{GeV}<M_{h}<127 \mathrm{GeV}$ from ATLAS and CMS collaborations of LHC [1,2]. In split SUSY, due to large $M_{S}$, $\log \left(m_{\tilde{f}}^{2} / m_{t}^{2}\right) \gg 1$ will spoil the convergence of the traditional loop expansion in evaluating the SUSY effects of Higgs boson self-energy. So in order to calculate mass of the SM-like Higgs boson, we use the RGE improved effective potential [62] which is employed in the NMSSMTools package [63] after we set $\lambda=\kappa \rightarrow 0$.

Note that the spin-independent (SI) dark matter-nucleon scattering rate is calculated with relevant parameters chosen as [64-67]: $f_{T_{u}}^{(p)}=0.023, f_{T_{d}}^{(p)}=0.032, f_{T_{u}}^{(n)}=0.017, f_{T_{d}}^{(n)}=0.041$ and $f_{T_{s}}^{(p)}=f_{T_{s}}^{(n)}=0.020$. We take into account all the contributions known so far (including QCD corrections) in our calculation of the scattering rate. The value of $f_{T_{s}}$ is taken from the recent lattice simulation results [68-70].

It is instructive to compare the dark matter constraints for non-universal and universal gaugino scenarios. Results for the universal gaugino scenario are taken from our previous work [21]. In figures 2 and 3 we show the samples surviving the constraints (1-4), where the green ' $X$ ' and red ' $\triangle$ ' denote respectively the samples allowed and excluded by the gauge coupling unification requirement. For these results we have the following discussions:

- We can see from figure 2 that increasing $M_{S}$ tends to slightly relax the gauge coupling unification constraints in non-universal gaugino scenario, in contrast to universal gaugino scenario where increasing $M_{S}$ tends to spoil the gauge coupling unification.

- For both scenarios, a strip corresponding to the higgsino dark matter with mass range from 1.0 to $1.3 \mathrm{TeV}$ can always survive the combined constraints of gauge coupling unification and dark matter direct detection (except $M_{S}>200 \mathrm{TeV}$ in universal gaugino scenario which is not preferred by gauge coupling unification requirement). 

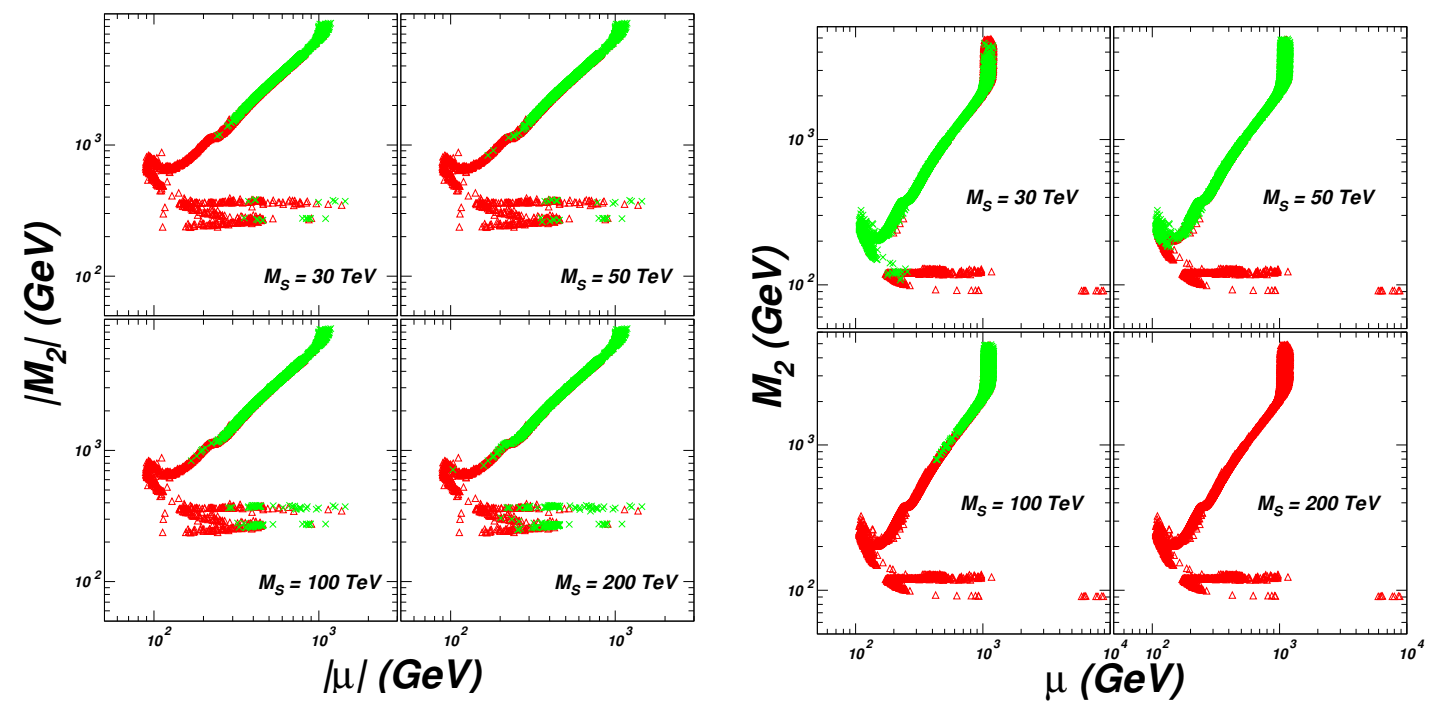

Figure 2. The scatter plots of the $\left(\mu, M_{2}\right)$ parameter space satisfying constraints (1-4) including dark matter relic density. The green ' $X$ ' (red ' $\triangle$ ') can (cannot) achieve the gauge coupling unification. The left panel is for the non-universal gaugino scenario proposed in this work while the right panel is for the universal gaugino scenario studied in our previous work [21].

This is the well known fact that higgsino at about $1.2 \mathrm{TeV}$ can be a viable dark matter candidate.

- Outside the strip of higgsino dark matter, almost all the survived points will be covered by XEON1T in both scenarios. An interesting exception occurs in nonuniversal gaugino scenario, where a tiny strip at about $50 \mathrm{GeV}$ cannot be covered by XEON1T and such a strip enlarges as $M_{S}$ increases. However, a careful analysis indicates that this strip corresponds to a bino dark matter. Although the gaugino mass ratio $M_{1}: M_{2}: M_{3}=1: 6: 12$ is no longer valid at the weak scale in split SUSY, the RGE running in general will not change significantly the mass ratio for a not too large $M_{S}$. So we can estimate that this strip corresponds to a gluino below $700 \mathrm{GeV}$. The current preliminary limits on gluino mass using $20 \mathrm{fb}^{-1}$ of $8 \mathrm{TeV}$ data are $M_{\tilde{g}}=1350 \mathrm{GeV}([74])$ and $M_{\tilde{g}}=1200 \mathrm{GeV}$ ([75]) assuming a massless neutralino for mini-split SUSY [76]. So this tiny strip should have been ruled out by the LHC data.

So we can see that a neutralino dark matter below about $1.0 \mathrm{TeV}$ will be fully covered by XENON1T for both non-universal and universal gaugino scenarios in split SUSY. We checked that our numerical calculation results are not sensitive to the sign of $\mu$, except that for the minus sign a very small part of parameter space (called blind spots) can survive all the constraints including the future XENON1T limits [73].

\section{Conclusion}

In this work we proposed to achieve the hierarchy of sparticle spectrum in split SUSY from the gap between the GUT scale and the Planck scale. We built a split SUSY model 

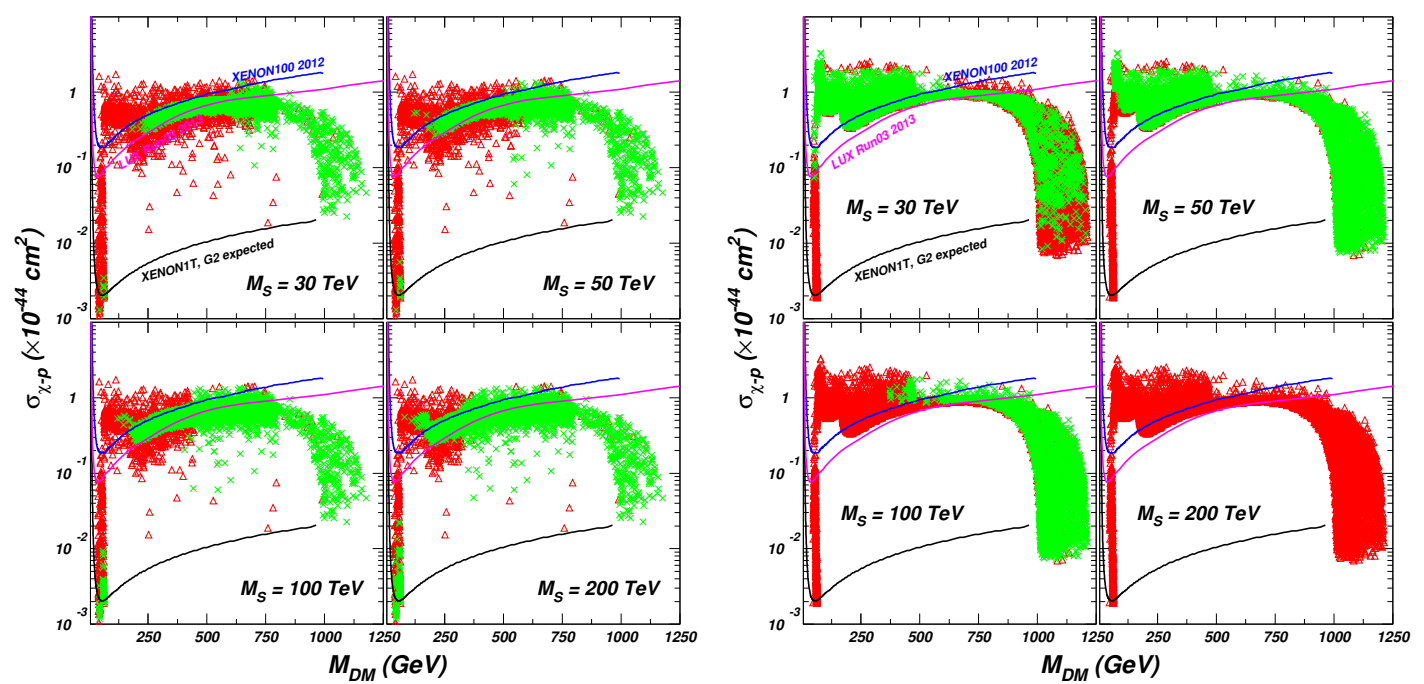

Figure 3. Same as figure 2, but showing the spin-independent cross section of dark matter scattering off the nucleon. The curves denote the limits from XENON100(2012) and LUX as well as the future XENON1T sensitivity.

(which gives non-universal gaugino masses) with proper high dimensional operators in the framework of $\mathrm{SO}(10)$ GUT. Based on a calculation of two-loop beta functions for gauge couplings (taking into account all weak scale threshold corrections), we checked the gauge coupling unification and dark matter constraints. We found that our scenario can achieve the gauge coupling unification and satisfy the dark matter constraints in some part of parameter space. We also examined the sensitivity of the future XENON1T experiment and found that the currently allowed parameter space in our scenario can be covered for a neutralino dark matter below about $1.0 \mathrm{TeV}$.

\section{Acknowledgments}

We are very grateful to the referee for useful comments. This work was supported by the Natural Science Foundation of China under grant numbers 11105124, 11105125, 11275245, 10821504, 11135003, 11375001, 11172008 and Ri-Xin Foundation of BJUT.

\section{A The two-loop beta function for gauge couplings}

In this work, we adopt the method in [77-79] to calculate the two-loop beta functions for three gauge couplings in the split SUSY, taking into account the weak scale threshold corrections. Our result is in agreement with $[17,19]$. The notations for the two-loop RGE can be found in the appendix of our previous work [21]. The one-loop beta functions for the three gauge couplings are given by

$$
\begin{aligned}
& b_{3}=-7 \theta\left(\mu-M_{Z}\right)+2 \theta\left(\mu-M_{\tilde{g}}\right)+2 \theta\left(\mu-M_{S}\right), \\
& b_{2}=-\frac{19}{6} \theta\left(\mu-M_{Z}\right)+\frac{4}{3} \theta\left(\mu-M_{\tilde{W}}\right)+\frac{2}{3} \theta\left(\mu-M_{\tilde{H}}\right)+\frac{13}{6} \theta\left(\mu-M_{S}\right), \\
& b_{1}=\frac{41}{10} \theta\left(\mu-M_{Z}\right)+\frac{2}{5} \theta\left(\mu-M_{\tilde{H}}\right)+\frac{21}{10} \theta\left(\mu-M_{S}\right),
\end{aligned}
$$


with the step function defined as

$$
\theta(x)= \begin{cases}1, & x \geq 0 \\ 0, & x<0\end{cases}
$$

The 2-loop RGE for $\mathrm{SU}(3)_{c}, \mathrm{SU}(2)_{L}, \mathrm{U}(1)_{Y}$ gauge couplings $\left(g_{3}, g_{2}, g_{1}\right.$, respectively) are given by

$$
\frac{d}{d \ln E} g_{i}=\frac{b_{i}}{(4 \pi)^{2}} g_{i}^{3}+\frac{g_{i}^{3}}{(4 \pi)^{4}}\left[\sum_{j} B_{i j} g_{j}^{2}-\sum_{a=u, d, e} d_{i}^{a} \operatorname{Tr}\left(h^{a \dagger} h^{a}\right)-d_{W}\left(\tilde{g}_{u}^{2}+\tilde{g}_{d}^{2}\right)-d_{B}\left(\tilde{g}_{u}^{\prime 2}+\tilde{g}_{d}^{\prime 2}\right)\right]
$$

with the $\mathrm{U}(1)_{Y}$ normalization $g_{1}^{2}=\frac{5}{3}\left(g_{Y}\right)^{2}$.

The two-loop beta functions for gauge couplings are given by

$$
\begin{aligned}
B_{i j}= & \theta\left(\mu-M_{Z}\right)\left(\begin{array}{ccc}
\frac{199}{50} & \frac{27}{10} & \frac{44}{5} \\
\frac{9}{10} & \frac{35}{6} & 12 \\
\frac{11}{10} & \frac{9}{2} & -26
\end{array}\right)+\theta\left(\mu-M_{2}\right)\left(\begin{array}{ccc}
0 & 0 & 0 \\
0 & \frac{64}{3} & 0 \\
0 & 0 & 0
\end{array}\right)+\theta\left(\mu-M_{3}\right)\left(\begin{array}{ccc}
0 & 0 & 0 \\
0 & 0 & 0 \\
0 & 0 & 48
\end{array}\right) \\
& +\theta\left(\mu-M_{\tilde{H}}\right)\left(\begin{array}{ccc}
\frac{9}{50} & \frac{9}{10} & 0 \\
\frac{3}{10} & \frac{49}{6} & 0 \\
0 & 0 & 0
\end{array}\right)+\theta\left(\mu-M_{S}\right)\left(\begin{array}{ccc}
\frac{19}{5} & \frac{9}{5} & \frac{44}{5} \\
\frac{3}{5} & -\frac{31}{3} & 12 \\
\frac{11}{10} & \frac{9}{2} & -8
\end{array}\right) .
\end{aligned}
$$

Similarly, we have

$$
\begin{aligned}
d^{u} & =\theta\left(\mu-M_{Z}\right)\left(\frac{17}{10}, \frac{3}{2}, 2\right)+\theta\left(\mu-M_{S}\right)\left(\frac{7}{2}, \frac{9}{2}, 2\right), \\
d^{d} & =\theta\left(\mu-M_{Z}\right)\left(\frac{1}{2}, \frac{3}{2}, 2\right)+\theta\left(\mu-M_{S}\right)\left(\frac{23}{10}, \frac{9}{2}, 2\right), \\
d^{e} & =\theta\left(\mu-M_{Z}\right)\left(\frac{3}{2}, \frac{1}{2}, 0\right)+\theta\left(\mu-M_{S}\right)\left(\frac{21}{10}, \frac{3}{2}, 0\right), \\
d^{W} & =\left(\frac{9}{20}, \frac{11}{4}, 0\right) \theta\left(\mu-\max \left(M_{2}, M_{\tilde{H}}\right)\right)+\theta\left(\mu-M_{S}\right)\left(-\frac{9}{20},-\frac{11}{4}, 0\right), \\
d^{B} & =\left(\frac{3}{20}, \frac{1}{4}, 0\right) \theta\left(\mu-\max \left(M_{1}, M_{\tilde{H}}\right)\right)+\theta\left(\mu-M_{S}\right)\left(-\frac{9}{20},-\frac{11}{4}, 0\right) .
\end{aligned}
$$

The one-loop renormalization group equations for Yukawa couplings below the $M_{S}$ scale can be written as

$$
\begin{aligned}
16 \pi^{2} \frac{d}{d t} h^{u} & =h^{u}\left[-3 c_{i}^{u} g_{i}^{2}+c_{T}^{u} T+c_{S_{1}}^{u} S_{1}+c_{S_{2}}^{u} S_{2}+\frac{3}{2}\left(h^{u \dagger} h^{u}-h^{d \dagger} h^{d}\right)\right], \\
16 \pi^{2} \frac{d}{d t} h^{d} & =h^{d}\left[-3 c_{i}^{d} g_{i}^{2}+c_{T}^{d} T+c_{S_{1}}^{d} S_{1}+c_{S_{2}}^{d} S_{2}+\frac{3}{2}\left(h^{d \dagger} h^{d}-h^{u \dagger} h^{u}\right)\right], \\
16 \pi^{2} \frac{d}{d t} h^{e} & =h^{e}\left[-3 c_{i}^{e} g_{i}^{2}+c_{T}^{e} T T+c_{S_{1}}^{e} S_{1}+c_{S_{2}}^{e} S_{2}+\frac{3}{2} h^{e \dagger} h^{e}\right],
\end{aligned}
$$

with

$$
T=\operatorname{Tr}\left(3 h^{u \dagger} h^{u}+3 h^{d \dagger} h^{d}+h^{e \dagger} h^{e}\right), S_{1}=\frac{1}{2}\left[\left(\tilde{g}_{u}^{\prime}\right)^{2}+\left(\tilde{g}_{d}^{\prime}\right)^{2}\right], S_{2}=\frac{3}{2}\left(\tilde{g}_{u}^{2}+\tilde{g}_{d}^{2}\right) .
$$


Above $M_{S}$, we will recover the MSSM result and the one-loop RGE for Yukawa-type interactions in the superpotential are well known to be

$$
\begin{aligned}
16 \pi^{2} \frac{d}{d t} \lambda^{u} & =\lambda^{u}\left[-2 c_{i}^{u} g_{i}^{2}+3 \operatorname{Tr}\left(\lambda^{u \dagger} \lambda^{u}\right)+3 \lambda^{u \dagger} \lambda^{u}+\lambda^{d \dagger} \lambda^{d}\right], \\
16 \pi^{2} \frac{d}{d t} \lambda^{d} & =\lambda^{d}\left[-2 c_{i}^{d} g_{i}^{2}+\operatorname{Tr}\left(3 \lambda^{d \dagger} \lambda^{d}+\lambda^{e \dagger} \lambda^{e}\right)+\lambda^{u \dagger} \lambda^{u}+3 \lambda^{d \dagger} \lambda^{d}\right], \\
16 \pi^{2} \frac{d}{d t} \lambda^{e} & =\lambda^{e}\left[-2 c_{i}^{e} g_{i}^{2}+\operatorname{Tr}\left(3 \lambda^{d \dagger} \lambda^{d}+\lambda^{e \dagger} \lambda^{e}\right)+3 \lambda^{e \dagger} \lambda^{e}\right],
\end{aligned}
$$

with

$$
c_{i}^{u}=\left(\frac{13}{30}, \frac{3}{2}, \frac{8}{3}\right), c_{i}^{d}=\left(\frac{7}{30}, \frac{3}{2}, \frac{8}{3}\right), c_{i}^{e}=\left(\frac{9}{10}, \frac{3}{2}, 0\right) .
$$

The one-loop Yukawa couplings for $c_{i}^{u}, c_{i}^{d}, c_{i}^{e}$ are calculated to be

$$
\left(\begin{array}{c}
c_{i}^{u} \\
c_{i}^{d} \\
c_{i}^{e}
\end{array}\right)=\theta\left(\mu-M_{Z}\right)\left(\begin{array}{ccc}
\frac{17}{60} & \frac{3}{4} & \frac{8}{3} \\
\frac{1}{12} & \frac{3}{4} & \frac{8}{3} \\
\frac{3}{4} & \frac{3}{4} & 0
\end{array}\right)+\theta\left(\mu-M_{S}\right)\left(\begin{array}{ccc}
\frac{3}{20} & \frac{3}{4} & 0 \\
\frac{3}{20} & \frac{3}{4} & 0 \\
\frac{3}{20} & \frac{3}{4} & 0
\end{array}\right)
$$

All terms are set to zero above $M_{S}$. Besides, we have

$$
\begin{aligned}
\left(\begin{array}{ccc}
c_{T}^{u} & c_{S_{1}}^{u} & c_{S_{2}}^{u} \\
c_{T}^{d} & c_{S_{1}}^{d} & c_{S_{1}}^{d} \\
c_{T}^{e} & c_{S_{1}}^{e} & c_{S_{2}}^{e}
\end{array}\right)= & \theta\left(\mu-M_{Z}\right)\left(\begin{array}{lll}
1 & 0 & 0 \\
1 & 0 & 0 \\
1 & 0 & 0
\end{array}\right)+\theta\left(\mu-\max \left(M_{2}, M_{\tilde{H}}\right)\right)\left(\begin{array}{lll}
0 & 0 & 1 \\
0 & 0 & 1 \\
0 & 0 & 1
\end{array}\right) \\
& +\theta\left(\mu-\max \left(M_{1}, M_{\tilde{H}}\right)\right)\left(\begin{array}{lll}
0 & 1 & 0 \\
0 & 1 & 0 \\
0 & 1 & 0
\end{array}\right)
\end{aligned}
$$

and all set to zero above $M_{S}$.

One loop RGE for gaugino couplings $\tilde{g}_{u}, \tilde{g}_{u}^{\prime}, \tilde{g}_{d}, \tilde{g}_{d}^{\prime}$ below $M_{S}$ are given as (with the gaugino relation $M_{1}<M_{2}$ )

- Between $\left[\max \left(M_{2}, M_{\tilde{H}}\right), M_{S}\right]$, the RGE for $\tilde{g}_{u, d}$ are given by

$$
\begin{aligned}
16 \pi^{2} \frac{d}{d t} \tilde{g}_{u}= & -3 \tilde{g}_{u} c_{i}^{u} g_{i}^{2}+\frac{5}{4} \tilde{g}_{u}^{3}-\frac{1}{2} \tilde{g}_{u} \tilde{g}_{d}^{2} \\
& +\frac{1}{4} \tilde{g}_{u} \tilde{g}_{u}^{\prime 2}+\tilde{g}_{d} \tilde{g}_{d}^{\prime} \tilde{g}_{u}^{\prime}+\tilde{g}_{u}\left(T+c_{S_{1}} S_{1}+c_{S_{2}} S_{2}\right) \\
16 \pi^{2} \frac{d}{d t} \tilde{g}_{d}= & -3 \tilde{g}_{d} c_{i}^{d} g_{i}^{2}+\frac{5}{4} \tilde{g}_{d}^{3}-\frac{1}{2} \tilde{g}_{d} \tilde{g}_{u}^{2} \\
& +\frac{1}{4} \tilde{g}_{d} \tilde{g}_{d}^{\prime 2}+\tilde{g}_{u} \tilde{g}_{u}^{\prime} \tilde{g}_{d}^{\prime}+\tilde{g}_{d}\left(T+c_{S_{1}} S_{1}+c_{S_{2}} S_{2}\right)
\end{aligned}
$$

with the coefficient

$$
c_{i}^{u, d}=\left(\frac{3}{20}, \frac{11}{4}, 0\right), \quad c_{S_{1}}=c_{S_{2}}=1 .
$$

Below $\max \left(M_{2}, M_{\tilde{H}}\right)$, the coupling are switched off. 
- Between $\left[\max \left(M_{2}, M_{\tilde{H}}\right), M_{S}\right]$, the RGE for $\tilde{g}_{u, d}$ are given by

$$
\begin{aligned}
16 \pi^{2} \frac{d}{d t} \tilde{g}_{u}^{\prime}= & -3 \tilde{g}_{u}^{\prime} \tilde{c}_{i}^{u} g_{i}^{2}+\frac{3}{4} \tilde{g}_{u}^{\prime 3}+\frac{3}{2} \tilde{g}_{u}^{\prime} \tilde{g}_{d}^{\prime 2} \\
& +\frac{3}{4} \tilde{g}_{u}^{\prime} \tilde{g}_{u}^{2}+3 \tilde{g}_{d}^{\prime} \tilde{g}_{d} \tilde{g}_{u}+\tilde{g}_{u}^{\prime}\left(T+c_{S_{1}} S_{1}+c_{S_{2}} S_{2}\right) \\
16 \pi^{2} \frac{d}{d t} \tilde{g}_{d}^{\prime}= & -3 \tilde{g}_{d}^{\prime} \tilde{c}_{i}^{d} g_{i}^{2}+\frac{3}{4} \tilde{g}_{d}^{\prime 3}+\frac{3}{2} \tilde{g}_{d}^{\prime} \tilde{g}_{u}^{\prime 2} \\
& +\frac{3}{4} \tilde{g}_{d}^{\prime} \tilde{g}_{d}^{2}+3 \tilde{g}_{u}^{\prime} \tilde{g}_{u} \tilde{g}_{d}+\tilde{g}_{d}^{\prime}\left(T+c_{S_{1}} S_{1}+c_{S_{2}} S_{2}\right)
\end{aligned}
$$

with the coefficient

$$
\tilde{c}_{i}^{u, d}=\left(\frac{3}{20}, \frac{3}{4}, 0\right), \quad c_{S_{1}}=c_{S_{2}}=1 .
$$

Between $\left[\max \left(M_{1}, M_{\tilde{H}}\right), \max \left(M_{2}, M_{\tilde{H}}\right)\right]$, the RGE reads

$$
\begin{aligned}
& 16 \pi^{2} \frac{d}{d t} \tilde{g}_{u}^{\prime}=-3 \tilde{g}_{u}^{\prime} \tilde{c}_{i}^{u} g_{i}^{2}+\frac{3}{4} \tilde{g}_{u}^{\prime 3}+\frac{3}{2} \tilde{g}_{u}^{\prime} \tilde{g}_{d}^{\prime 2}+\tilde{g}_{u}^{\prime}\left(T+c_{S_{1}} S_{1}+c_{S_{2}} S_{2}\right), \\
& 16 \pi^{2} \frac{d}{d t} \tilde{g}_{d}^{\prime}=-3 \tilde{g}_{d}^{\prime} \tilde{c}_{i}^{d} g_{i}^{2}+\frac{3}{4} \tilde{g}_{d}^{\prime 3}+\frac{3}{2} \tilde{g}_{d}^{\prime} \tilde{g}_{u}^{\prime 2}+\tilde{g}_{d}^{\prime}\left(T+c_{S_{1}} S_{1}+c_{S_{2}} S_{2}\right),
\end{aligned}
$$

with the coefficient

$$
\tilde{c}_{i}^{u, d}=\left(\frac{3}{20}, \frac{3}{4}, 0\right), \quad c_{S_{1}}=1, \quad c_{S_{2}}=0 .
$$

Below $\max \left(M_{1}, M_{\tilde{H}}\right)$, the coupling are switched off.

Open Access. This article is distributed under the terms of the Creative Commons Attribution License (CC-BY 4.0), which permits any use, distribution and reproduction in any medium, provided the original author(s) and source are credited.

\section{References}

[1] ATLAS collaboration, Combined search for the Standard Model Higgs boson using up to $4.9 \mathrm{fb}^{-1}$ of $p p$ collision data at $\sqrt{\mathrm{s}}=7$ TeV with the ATLAS detector at the LHC, Phys. Lett. B 710 (2012) 49 [arXiv:1202.1408] [INSPIRE].

[2] CMS collaboration, Combined results of searches for the standard model Higgs boson in pp collisions at $\sqrt{s}=7$ TeV, Phys. Lett. B 710 (2012) 26 [arXiv:1202.1488] [INSPIRE].

[3] H. Georgi and S.L. Glashow, Unity of all elementary particle forces, Phys. Rev. Lett. 32 (1974) 438 [InSPIRE].

[4] J.R. Ellis, S. Kelley and D.V. Nanopoulos, Precision LEP data, supersymmetric GUTs and string unification, Phys. Lett. B 249 (1990) 441 [INSPIRE].

[5] J.R. Ellis, S. Kelley and D.V. Nanopoulos, Probing the desert using gauge coupling unification, Phys. Lett. B 260 (1991) 131 [INSPIRE].

[6] U. Amaldi, W. de Boer and H. Furstenau, Comparison of grand unified theories with electroweak and strong coupling constants measured at LEP, Phys. Lett. B 260 (1991) 447 [INSPIRE]. 
[7] P. Langacker and M.-x. Luo, Implications of precision electroweak experiments for $M_{t}, \rho_{0}$, $\sin ^{2} \theta_{W}$ and grand unification, Phys. Rev. D 44 (1991) 817 [INSPIRE].

[8] M.B. Einhorn and D.R.T. Jones, The weak mixing angle and unification mass in supersymmetric SU(5), Nucl. Phys. B 196 (1982) 475 [InSPIRE].

[9] W.J. Marciano and G. Senjanović, Predictions of supersymmetric grand unified theories, Phys. Rev. D 25 (1982) 3092 [inSPIRE].

[10] ATLAS collaboration, Search for squarks and gluinos using final states with jets and missing transverse momentum with the ATLAS detector in $\sqrt{s}=7 \mathrm{TeV}$ proton-proton collisions, Phys. Lett. B 710 (2012) 67 [arXiv:1109.6572] [INSPIRE].

[11] ATLAS collaboration, Search for squarks and gluinos with the ATLAS detector in final states with jets and missing transverse momentum using $4.7 \mathrm{fb}^{-1}$ of $\sqrt{\mathrm{s}}=7 \mathrm{TeV}$ proton-proton collision data, Phys. Rev. D 87 (2013) 012008 [arXiv: 1208.0949] [INSPIRE].

[12] CMS collaboration, Search for supersymmetry at the LHC in events with jets and missing transverse energy, Phys. Rev. Lett. 107 (2011) 221804 [arXiv:1109.2352] [INSPIRE].

[13] CMS collaboration, Search for supersymmetry in hadronic final states using MT2 in pp collisions at $\sqrt{s}=7 \mathrm{TeV}$, JHEP 10 (2012) 018 [arXiv:1207.1798] [INSPIRE].

[14] J.-J. Cao, Z.-X. Heng, J.M. Yang, Y.-M. Zhang and J.-Y. Zhu, A SM-like Higgs near $125 \mathrm{GeV}$ in low energy SUSY: a comparative study for MSSM and NMSSM, JHEP 03 (2012) 086 [arXiv: 1202.5821] [INSPIRE].

[15] J. Cao, Z. Heng, J.M. Yang and J. Zhu, Status of low energy SUSY models confronted with the LHC $125 \mathrm{GeV}$ Higgs data, JHEP 10 (2012) 079 [arXiv:1207.3698] [INSPIRE].

[16] N. Arkani-Hamed and S. Dimopoulos, Supersymmetric unification without low energy supersymmetry and signatures for fine-tuning at the LHC, JHEP 06 (2005) 073 [hep-th/0405159] [INSPIRE].

[17] G.F. Giudice and A. Romanino, Split supersymmetry, Nucl. Phys. B 699 (2004) 65 [Erratum ibid. B 706 (2005) 65-89] [hep-ph/0406088] [INSPIRE].

[18] N. Arkani-Hamed, S. Dimopoulos, G.F. Giudice and A. Romanino, Aspects of split supersymmetry, Nucl. Phys. B 709 (2005) 3 [hep-ph/0409232] [INSPIRE].

[19] G.F. Giudice and A. Strumia, Probing high-scale and split supersymmetry with Higgs mass measurements, Nucl. Phys. B 858 (2012) 63 [arXiv:1108.6077] [InSPIRE].

[20] A. Arvanitaki, N. Craig, S. Dimopoulos and G. Villadoro, Mini-split, JHEP 02 (2013) 126 [arXiv: 1210.0555$]$ [INSPIRE].

[21] F. Wang, W. Wang and J.M. Yang, Split supersymmetry under GUT and current dark matter constraints, Eur. Phys. J. C 74 (2014) 3121 [arXiv:1310.1750] [INSPIRE].

[22] A.H. Chamseddine, R.L. Arnowitt and P. Nath, Locally supersymmetric grand unification, Phys. Rev. Lett. 49 (1982) 970 [INSPIRE].

[23] H.P. Nilles, Dynamically broken supergravity and the hierarchy problem, Phys. Lett. B 115 (1982) 193 [INSPIRE].

[24] L.E. Ibáñez, Locally supersymmetric SU(5) grand unification, Phys. Lett. B 118 (1982) 73 [INSPIRE].

[25] R. Barbieri, S. Ferrara and C.A. Savoy, Gauge models with spontaneously broken local supersymmetry, Phys. Lett. B 119 (1982) 343 [INSPIRE]. 
[26] H.P. Nilles, M. Srednicki and D. Wyler, Weak interaction breakdown induced by supergravity, Phys. Lett. B 120 (1983) 346 [inSPIRE].

[27] J.R. Ellis, D.V. Nanopoulos and K. Tamvakis, Grand unification in simple supergravity, Phys. Lett. B 121 (1983) 123 [inSPIRE].

[28] J.R. Ellis, J.S. Hagelin, D.V. Nanopoulos and K. Tamvakis, Weak symmetry breaking by radiative corrections in broken supergravity, Phys. Lett. B 125 (1983) 275 [INSPIRE].

[29] L.J. Hall, J.D. Lykken and S. Weinberg, Supergravity as the messenger of supersymmetry breaking, Phys. Rev. D 27 (1983) 2359 [inSPIRE].

[30] N. Ohta, Grand unified theories based on local supersymmetry, Prog. Theor. Phys. 70 (1983) 542 [INSPIRE].

[31] T. Li and D.V. Nanopoulos, Generalizing minimal supergravity, Phys. Lett. B 692 (2010) 121 [arXiv:1002.4183] [INSPIRE].

[32] C. Balázs, T. Li, D.V. Nanopoulos and F. Wang, Supersymmetry breaking scalar masses and trilinear soft terms in generalized minimal supergravity, JHEP 09 (2010) 003 [arXiv: 1006.5559] [INSPIRE].

[33] F. Wang, Supersymmetry breaking scalar masses and trilinear soft terms from high-dimensional operators in $E_{6}$ SUSY GUT, Nucl. Phys. B 851 (2011) 104 [arXiv: 1103.0069] [INSPIRE].

[34] J.R. Ellis, K. Enqvist, D.V. Nanopoulos and K. Tamvakis, Gaugino masses and grand unification, Phys. Lett. B 155 (1985) 381 [InSPIRE].

[35] M. Drees, Phenomenological consequences of $N=1$ supergravity theories with nonminimal kinetic energy terms for vector superfields, Phys. Lett. B 158 (1985) 409 [INSPIRE].

[36] B. Ananthanarayan and P.N. Pandita, Sparticle mass spectrum in grand unified theories, Int. J. Mod. Phys. A 22 (2007) 3229 [arXiv:0706.2560] [InSPIRE].

[37] S. Bhattacharya, A. Datta and B. Mukhopadhyaya, Non-universal gaugino masses: a signal-based analysis for the Large Hadron Collider, JHEP 10 (2007) 080 [arXiv:0708.2427] [INSPIRE].

[38] S.P. Martin, Non-universal gaugino masses from non-singlet F-terms in non-minimal unified models, Phys. Rev. D 79 (2009) 095019 [arXiv:0903.3568] [INSPIRE].

[39] J. Chakrabortty and A. Raychaudhuri, A note on dimension-5 operators in GUTs and their impact, Phys. Lett. B 673 (2009) 57 [arXiv:0812.2783] [INSPIRE].

[40] S.P. Martin, Nonuniversal gaugino masses and seminatural supersymmetry in view of the Higgs boson discovery, Phys. Rev. D 89 (2014) 035011 [arXiv: 1312.0582] [INSPIRE].

[41] J.E. Younkin and S.P. Martin, Non-universal gaugino masses, the supersymmetric little hierarchy problem and dark matter, Phys. Rev. D 85 (2012) 055028 [arXiv:1201.2989] [INSPIRE].

[42] S. Jung and J.D. Wells, Gaugino physics of split supersymmetry spectra at the LHC and future proton colliders, Phys. Rev. D 89 (2014) 075004 [arXiv:1312.1802] [INSPIRE].

[43] I. Gogoladze, F. Nasir, Q. Shafi and C.S. Un, Nonuniversal gaugino masses and muon $g-2$, Phys. Rev. D 90 (2014) 035008 [arXiv: 1403.2337] [INSPIRE].

[44] M. Chakraborti, U. Chattopadhyay, S. Rao and D.P. Roy, Higgsino dark matter in nonuniversal gaugino mass models, Phys. Rev. D 91 (2015) 035022 [arXiv:1411.4517] [INSPIRE]. 
[45] R. Slansky, Group theory for unified model building, Phys. Rept. 79 (1981) 1 [InSPIRE].

[46] S.P. Martin and M.T. Vaughn, Regularization dependence of running couplings in softly broken supersymmetry, Phys. Lett. B 318 (1993) 331 [hep-ph/9308222] [INSPIRE].

[47] Super-Kamiokande collaboration, M. Miura, Search for nucleon decays in Super-Kamiokande, PoS(ICHEP 2010) 408.

[48] TITAND Working Group collaboration, Y. Suzuki et al., Multimegaton water Cherenkov detector for a proton decay search: TITAND (former name TITANIC), hep-ex/0110005 [INSPIRE].

[49] J. Hisano, D. Kobayashi, T. Kuwahara and N. Nagata, Decoupling can revive minimal supersymmetric SU(5), JHEP 07 (2013) 038 [arXiv: 1304.3651] [INSPIRE].

[50] J. Hisano, T. Kuwahara and N. Nagata, Grand unification in high-scale supersymmetry, Phys. Lett. B 723 (2013) 324 [arXiv: 1304.0343] [INSPIRE].

[51] N. Bernal, A. Djouadi and P. Slavich, The MSSM with heavy scalars, JHEP 07 (2007) 016.

[52] N. Bernal, Dark matter direct detection in the MSSM with heavy scalars, JCAP 08 (2009) 022 [arXiv:0905.4239] [inSPIRE].

[53] Planck collaboration, P.A.R. Ade et al., Planck 2013 results. XVI. Cosmological parameters, Astron. Astrophys. 571 (2014) A16 [arXiv:1303.5076] [INSPIRE].

[54] WMAP collaboration, J. Dunkley et al., Five-year Wilkinson Microwave Anisotropy Probe (WMAP) observations: likelihoods and parameters from the WMAP data, Astrophys. J. Suppl. 180 (2009) 306 [arXiv:0803.0586] [INSPIRE].

[55] XENON100 collaboration, E. Aprile et al., Dark matter results from 225 live days of XENON100 data, Phys. Rev. Lett. 109 (2012) 181301 [arXiv:1207.5988] [INSPIRE].

[56] LUX collaboration, D.S. Akerib et al., First results from the LUX dark matter experiment at the Sanford Underground Research Facility, Phys. Rev. Lett. 112 (2014) 091303 [arXiv: 1310.8214] [INSPIRE].

[57] P. Gondolo et al., DarkSUSY: Computing supersymmetric dark matter properties numerically, JCAP 07 (2004) 008 [astro-ph/0406204] [INSPIRE]; see http://www.physto.se/ edsjo/darksusy.

[58] F. Wang, W. Wang and J.M. Yang, Dark matter constraints on gaugino/Higgsino masses in split supersymmetry and their implications at colliders, Eur. Phys. J. C 46 (2006) 521 [hep-ph/0512133] [INSPIRE].

[59] G. Altarelli and R. Barbieri, Vacuum polarization effects of new physics on electroweak processes, Phys. Lett. B 253 (1991) 161 [INSPIRE].

[60] M.E. Peskin and T. Takeuchi, Estimation of oblique electroweak corrections, Phys. Rev. D 46 (1992) 381 [INSPIRE].

[61] ALEPH, DELPHI, L3, OPAL, SLD, LEP Electroweak Working Group, SLD Electroweak Group, SLD Heavy Flavour Group collaboration, S. Schael et al., Precision electroweak measurements on the $Z$ resonance, Phys. Rept. 427 (2006) 257 [hep-ex/0509008] [INSPIRE].

[62] M. Binger, Higgs boson mass in split supersymmetry at two-loops, Phys. Rev. D 73 (2006) 095001 [hep-ph/0408240] [INSPIRE]. 
[63] U. Ellwanger, J.F. Gunion and C. Hugonie, NMHDECAY: a Fortran code for the Higgs masses, couplings and decay widths in the NMSSM, JHEP 02 (2005) 066 [hep-ph/0406215] [INSPIRE].

[64] A. Djouadi and M. Drees, QCD corrections to neutralino nucleon scattering, Phys. Lett. B 484 (2000) 183 [hep-ph/0004205] [INSPIRE].

[65] G. Bélanger, F. Boudjema, A. Pukhov and A. Semenov, Dark matter direct detection rate in a generic model with MicrOMEGAs 2.2, Comput. Phys. Commun. 180 (2009) 747 [arXiv: 0803.2360] [INSPIRE].

[66] M.S. Carena, D. Garcia, U. Nierste and C.E.M. Wagner, Effective Lagrangian for the $\bar{t} b H^{+}$ interaction in the MSSM and charged Higgs phenomenology, Nucl. Phys. B 577 (2000) 88 [hep-ph/9912516] [INSPIRE].

[67] J. Hisano, K. Ishiwata and N. Nagata, Gluon contribution to the dark matter direct detection, Phys. Rev. D 82 (2010) 115007 [arXiv:1007.2601] [INSPIRE].

[68] H. Ohki et al., Nucleon sigma term and strange quark content from lattice QCD with exact chiral symmetry, Phys. Rev. D 78 (2008) 054502 [arXiv:0806.4744] [INSPIRE].

[69] MILC collaboration, D. Toussaint and W. Freeman, The strange quark condensate in the nucleon in $2+1$ flavor QCD, Phys. Rev. Lett. 103 (2009) 122002 [arXiv:0905.2432] [INSPIRE].

[70] J. Giedt, A.W. Thomas and R.D. Young, Dark matter, the CMSSM and lattice QCD, Phys. Rev. Lett. 103 (2009) 201802 [arXiv:0907.4177] [INSPIRE].

[71] N. Arkani-Hamed, S. Dimopoulos and S. Kachru, Predictive landscapes and new physics at a $T e V$, hep-th/0501082 [INSPIRE].

[72] R. Mahbubani and L. Senatore, The minimal model for dark matter and unification, Phys. Rev. D 73 (2006) 043510 [hep-ph/0510064] [INSPIRE].

[73] C. Cheung, L.J. Hall, D. Pinner and J.T. Ruderman, Prospects and blind spots for neutralino dark matter, JHEP 05 (2013) 100 [arXiv:1211.4873] [INSPIRE].

[74] ATLAS collaboration, Search for squarks and gluinos with the ATLAS detector in final states with jets and missing transverse momentum and $20.3 \mathrm{fb}^{-1}$ of $\sqrt{s}=8 \mathrm{TeV}$ proton-proton collision data, ATLAS-CONF-2013-047 (2013).

[75] CMS collaboration, Search for new physics in the multijets and missing momentum final state in proton-proton collisions at 8 TeV, CMS-PAS-SUS-13-012 (2013).

[76] T. Cohen et al., SUSY simplified models at 14, 33 and 100 TeV proton colliders, JHEP 04 (2014) 117 [arXiv:1311.6480] [INSPIRE].

[77] M.E. Machacek and M.T. Vaughn, Two loop renormalization group equations in a general quantum field theory. 1. Wave function renormalization, Nucl. Phys. B 222 (1983) 83 [INSPIRE].

[78] M.E. Machacek and M.T. Vaughn, Two loop renormalization group equations in a general quantum field theory. 2. Yukawa couplings, Nucl. Phys. B 236 (1984) 221 [inSPIRE].

[79] S.P. Martin and M.T. Vaughn, Two loop renormalization group equations for soft supersymmetry breaking couplings, Phys. Rev. D 50 (1994) 2282 [Erratum ibid. D 78 (2008) 039903] [hep-ph/9311340] [INSPIRE]. 